\title{
Research Paper \\ Psychometric Properties of Persian Version of Young-Rygh Avoidance Inventory
}

\section{Erfan Soleimani-Sefat ${ }^{1}$, Himman Sa'adati² ${ }^{*}$, Sorayya Azimian ${ }^{1}$, Shahin Amani ${ }^{3}$, Hiva Saleh-Manijeh ${ }^{1}$, Leila Leshni ${ }^{1}$}

1. MSc. of Rehabilitation Counseling, Department of Counseling, University of Social Welfare and Rehabilitation Sciences, Tehran, Iran.

2. PhD Student in Rehabilitation Counseling, Department of Counseling, Student Research Committee, University of Social Welfare and Rehabilitation Sciences, Tehran, Iran.

3. MSc. Student of Preschool, Department of Preschool, University of Social Welfare and Rehabilitation Sciences, Tehran, Iran.

Received: 17 Jun. 2016 Accepted: 26 Nov. 2016

Key words: Validity, Reliability, Factor analysis Avoidance Inventory
Ceftation: Soleimani-Sefat E, Sa'adati H, Azimian S, Amani Sh, Saleh-Manijeh H, Leshni L. [Psychometric Properties of Persian Version of Young-Rygh Avoidance Inventory (Persian)]. Iranian Journal of Psychiatry and Clinical Psychology. 2017; 23(2):232-243. https://doi.org/10.29252/nirp.ijpcp.23.2.232

https://doi.org/10.29252/nirp.ijpcp.23.2.232

\section{T}

\section{Extended Abstract}

\section{Introduction}

he term "schema" is being used in psychological texts since the early twentieth century, and it seems that Bartlett was the first one to mention the concept of schema in psychology and scope of memory. Schema therapy is an innovative approach and a combination of cognitive-behavioral therapy, the psychoanalytic object relations theory, attachment theory, and gestalt therapy that were established by Young et al. Since there is no questionnaire with proper validity and reliability to measure avoidance structure within the country, an instrument is needed to measure the amount of avoidance . Therefore, the present study aims to assess the validity and reliability of the Persian version of Young-Rygh Avoidance Inventory and analyze its factors.

\section{Method}

The current correlational study is of factor analysis type. Since the target is non-clinical population, the sample group

* Corresponding Author: Himman Sa'adati, PhD Student

Address: Department of Counseling, Student Research Committee, University of Social Welfare and Rehabilitation Sciences, Tehran, Iran. Tel: +98 (936) 3665106

E-mail: hemnsaadati@gmail.com 
consisted of 400 students from the University of Social Welfare and Rehabilitation Sciences studying in 2014-2015 academic year. The participants were selected using convenient sampling. They participated voluntarily in the study with the knowledge of research purposes. The inclusion criteria were non-usage of psychological drugs, no psychological treatments, and informed consent to participate in the study. The subjects who completed less than $10 \%$ of the items in the questionnaire were excluded from the statistical analysis. According to experts, 5-10 subjects were required for each item of the validity and reliability evaluation questionnaires. Thus, 400 individuals were initially selected that reduced to 382 people (114 males and 268 females) with a mean age of 24.6 years as 18 questionnaires were excluded from the statistical analysis . A sample group of Avoidance Questionnaire, Beck Depression Inventory-Second Edition, Beck Anxiety Inventory, Young Overcompensation Inventory and Acceptance and Action Questionnaire- Second Edition (AAQ-II) were considered in this study. The missing data were replaced by the mean of each group.

\section{Results}

KMO value obtained from the exploratory factor analysis equals to 0.79 and indicates the adequacy of the sample for analyzing the factors. The Bartlett level is also 6831.71, which is statistically significant $(\mathrm{P}<0.001)$. Factor analysis results show that 12 factors have been extracted, among which 8 factors, accounting for $54.92 \%$ of the total variance, could be interpreted. To improve the interpretability of these factors, Varimax rotation method was used. Extracted factors are as follows: isolation, substance abuse, denied dissatisfaction, rationality and extreme control, passively blocking troublesome excitement, psychotic symptoms, distraction, and denying internal experiences.

Questions having a factor coefficient less than 0.3 were excluded from the questionnaire, and the rotation of the factors showed that some factors, particularly factors that share common items, merged with other factors. The factor of intentionally not thinking about troublesome issues and the factor of denial of memories merged into denial of internal experiences, factor of anxiety downtime merged with factor of rationality and extreme control, and factor of avoidance through sleep, self-soothing and passive distraction merged with distraction factor.

Factor of avoidance from turbulent situation, which has only one item (37) in the main questionnaire, was not loaded on any factor and was deleted. Generally, 13 items were not loaded in any of the factors and were removed from statistical analysis. To assess the convergent-divergent validity of the Avoidance Questionnaire, Acceptance and Action
Questionnaire (AAQ-II), Beck Depression Inventory-Second Edition, Beck Anxiety Questionnaire, and the Young overcompensation inventory.

\section{Discussion}

After analyzing the factors of the avoidance questionnaire in the present study, eight factors extracted. To verify the reliability of the questionnaire, alpha and test-retest were used to determine inner consistency and time reliability of the questionnaires. The factor of passively blocking emotions had the lowest internal stability and the temporal reliability and factors of denial of dissatisfaction, rationality, and extreme control had the highest internal and temporal reliability, respectively. These results are consistent with the results of studies by Spranger et al. (2001) [19], Sheffield et al. (2009) [20], and Zargar et al. (2011) [22] in which internal reliability of the questionnaire were $0.78,0.67,0.70$, and 0.71 , respectively. The temporal reliability of the questionnaire was measured through the test-retest method, and the results indicate the appropriate reliability of the questionnaire.

In the present study, to check the criterion validity, correlation was used between avoidance questionnaire and four questionnaires of Acceptance and Action Questionnaires (AAQ-II), Beck Depression Inventory-Second Edition, Beck Anxiety Inventory, and the Extreme Compensation Questionnaire. Results obtained from the Pearson correlation test to assess convergent and reciprocal validity showed that the Avoidance Questionnaire has negative relationship with the Acceptance and Action Questionnaire and has a positive relationship with Extreme Anxiety and Extreme Compensation.

These results indicate appropriate criterion validity for the Avoidance Questionnaire. To the best of our knowledge, there is no research on the validity of Young-Rygh Avoidance Inventory, and studies using this questionnaire only assessed the internal reliability of the questionnaire by Cronbach's Alpha. In the present study, consistent studies that assessed the validity of maladaptive schema questionnaire have been used. The significant relationship between the Avoidance Questionnaire and Beck Depression Inventory-Second Edition and Beck's Anxiety Questionnaire is consistent with the results of studies by Divandari et al. (2009) [28] and Fata et al. (2005) [29]. Correlation of Avoidance Questionnaire with Young's Extreme Compensation Inventory was foreseeable as mentioned in the theoretical texts, and the strongest correlation was related to Young's Extreme Compensation Questionnaire. In fact, from a theoretical point of view, extreme compensation is 
one of the avoidance mechanisms; therefore, this relation can be explained.

The results of this study show that the Persian version of Young-Rygh Avoidance Inventory has appropriate reliability and validity. The results of the diagnostic factor analysis showed that eight factors were extracted from this questionnaire. The result of the confirmatory factor analysis indicates that the model is adequately fit. Also this questionnaire was found to have appropriate (internal and temporal) criterion validity and reliability and can be used for research purposes in the Iranian population. However, like any other study, the findings of this study should be construed within the limits of its constraints. Since student population, i.e., non-clinical population, was used in this study, the results of this study cannot be generalized to the clinical group . Thus, future studies should assess psychoanalytic characteristics of this questionnaire in the clinical population.

\section{Acknowledgments}

University of Social Welfare and Rehabilitation Sciences Student Research Committee has financially supported the present study.

\section{Conflict of Interest}

The authors declared no conflicts of interest. 


\title{
ويرَّى هاى روانسنجى نسخه فارسى يرسشنامه اجتناب يانتى و ريخى
}

\author{
عرفان سليمانىصفت'، "هيمن سعادتى'، ثريا عظيميان'، شهين امانى'، هيوا صالح منيجه'، ليلا لشنى' \\ ا - كارشناسى ارشد مشاوره توائبخشى، كروه مشاوره، دائشكاه علوم بهزيستى و توانبخشى، تهرانه ايران.

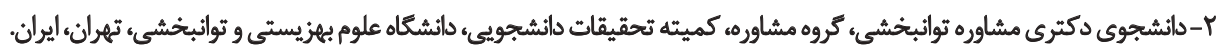

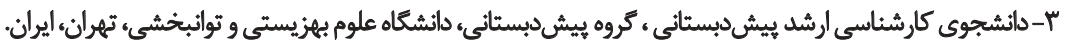

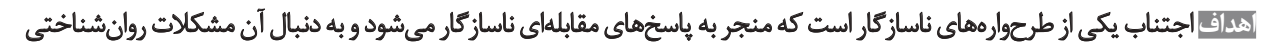

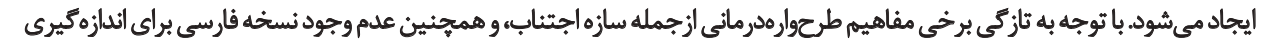

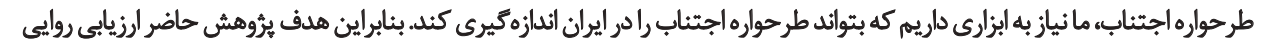

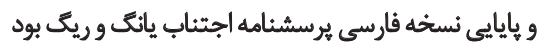

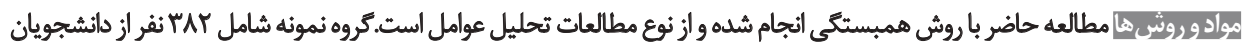

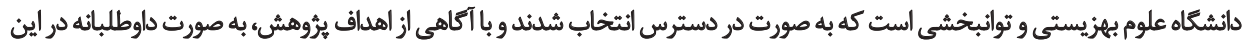

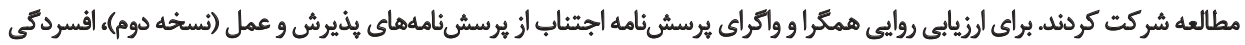

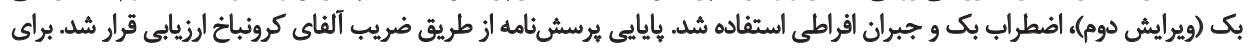

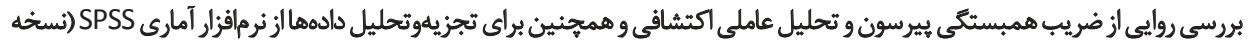
(T) استفاده شد.

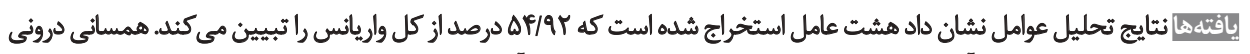

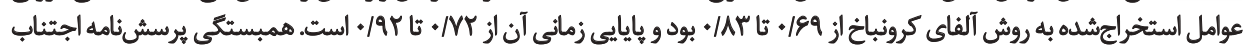

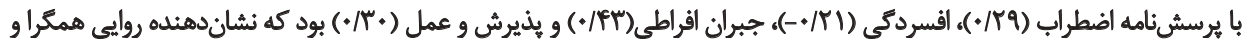

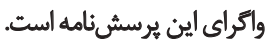

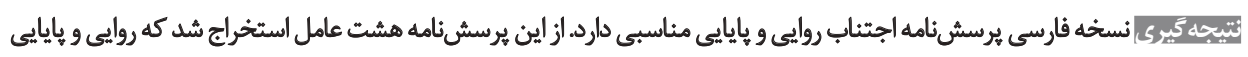
مئاسبي دارئد.
\end{abstract}

تاريخ دريافت:

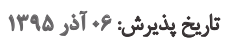

ميشود و بر اساس واقعيت يا تجربه شكل مى كيرد تا به افراد

dalo

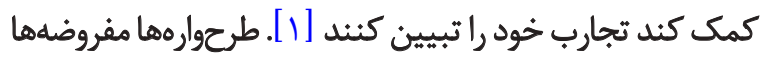

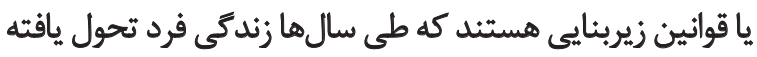

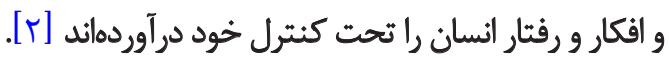

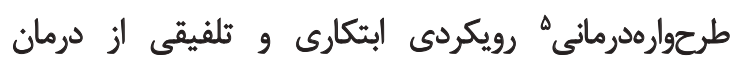

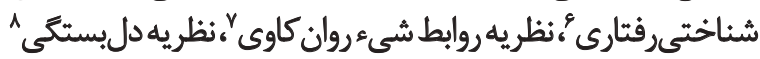

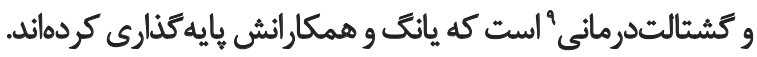

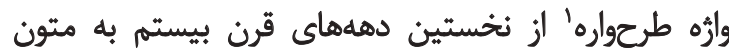

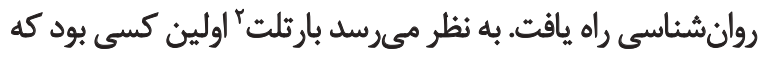

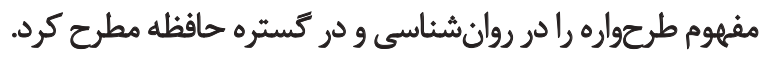

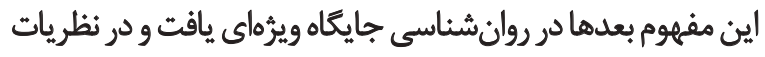

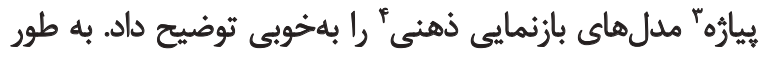

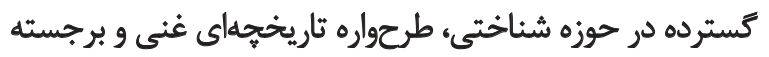

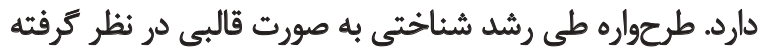

5. Schema therapy

6. Cognitive behavioral therapy

1. Schema

7. Psychoanalytic object relations theory

2. Bartlett

8. Attachment theory

3. Piaget

9. Gestalt therapy

4. Mental representation

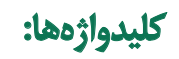

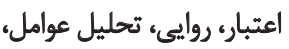

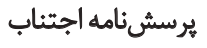

$$
\text { -. }
$$

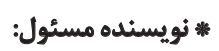

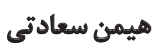
نشانىي: تهران، دانشكاه علوم بهزيستى و توانبخشى، كميثه تحقيقات دانشجويى، كروه مشاوره. تلفن: بست الكترونيكي: بلفي 
هحققان مختلف انواع راهبردهاي اجتئاب شناختى ازجمله

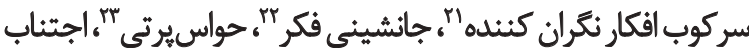

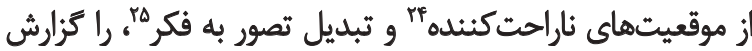

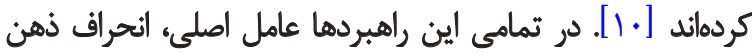

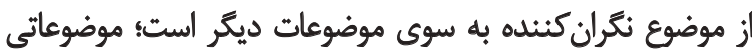

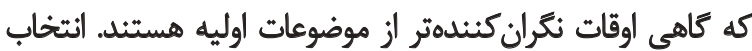

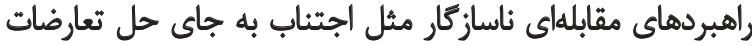

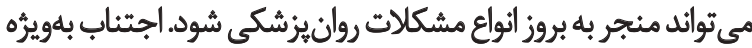

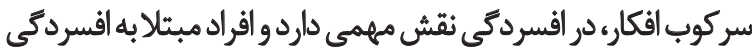

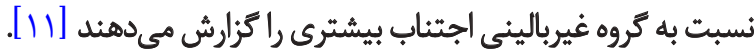

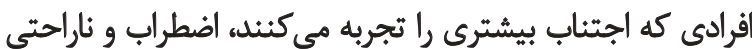

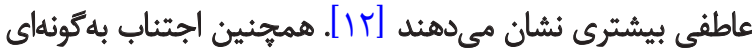

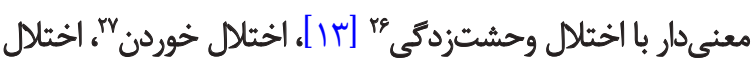

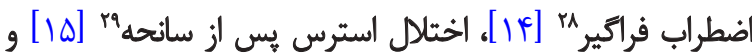
اختلال وسواس كندن مو [ع1 ] إرتباط دارد.

در همين راستا يانك و ريك در سال 1994 به منظور سنجش

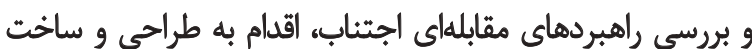

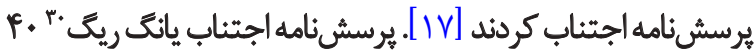

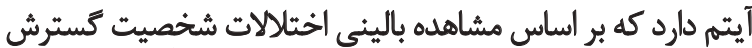

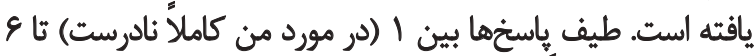

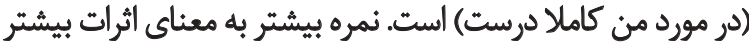

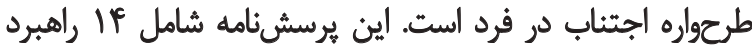

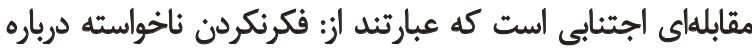

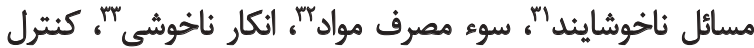

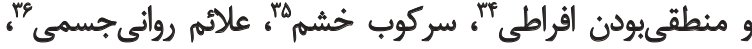

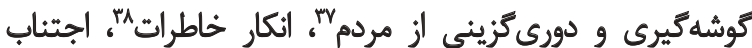

21. Suppressing worrisome thoughts

22. Substitution thought

23. Distraction

24. Avoidance of upsetting situations

25. Convert the image to thought

26. Panic disorder

27. Eating disorder

28. Generalized anxiety disorder

29. Posttraumatic stress disorder

30. Young-Rygh Avoidance Inventory (YRAI)

31. Intentionally not thinking about upsetting things

32. Substance abuse

33. Denial of unhappiness

34. Excessive rationality and contro

35. Suppression of anger

36. Psychosomatic symptoms

37. Withdrawal from people

38. Denial of memories

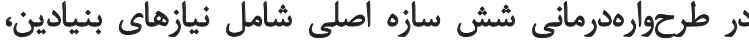

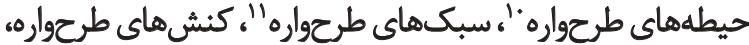

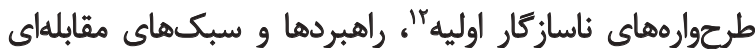

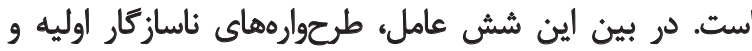

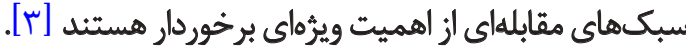

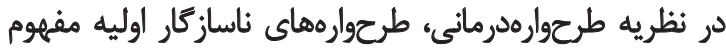

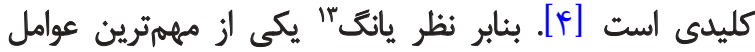

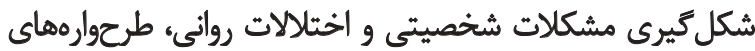

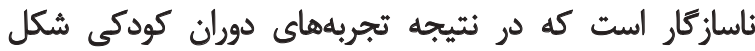

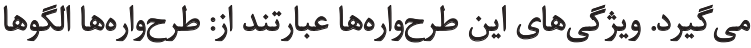

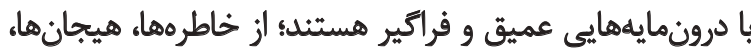

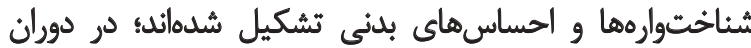

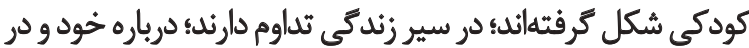

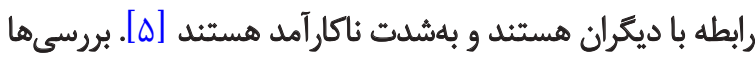

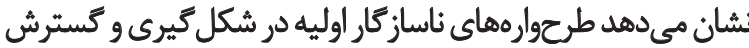

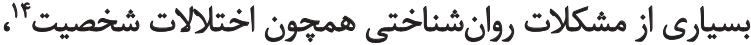

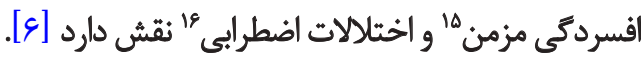

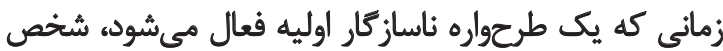

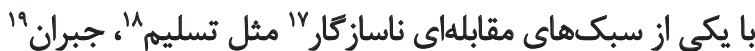

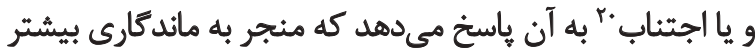

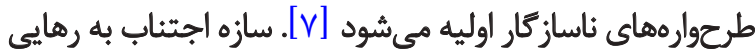

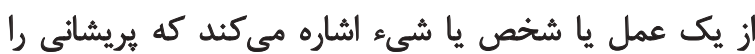

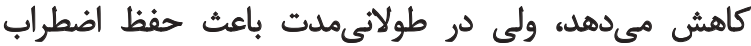

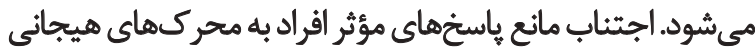

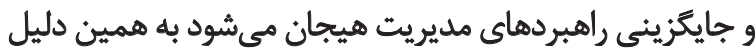
راهبردى كارآمدنيست.

طرحواره اجتناب شامل استفاده از رفتارهايى ميى فيود كه آكاهى

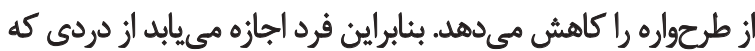

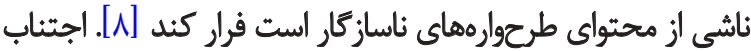

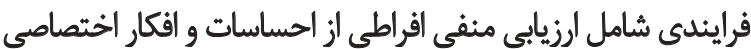

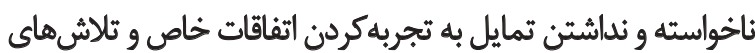

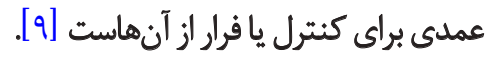

10. Schemas domains

11. Schema modes

12. Early maladaptive schemas

13. Yang

14. Personality disorders

15. Chronic depression

16. Anxiety disorders

17. Maladaptive coping styles

18. Submission

19. Compensation

20. Avoidance 
ارائهشده در طرحوارهدرمانى هم هم تواند در كروههاى بالينى

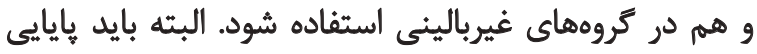

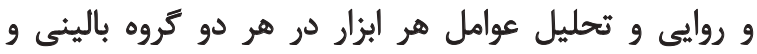

$$
\text { غيربالينى بررسى شود. }
$$

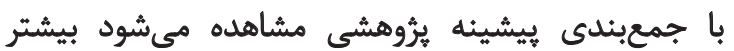

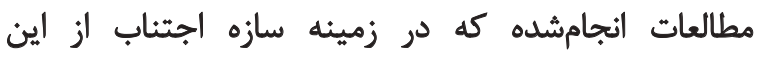

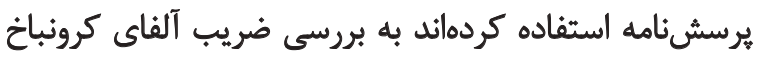

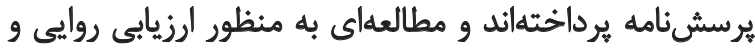

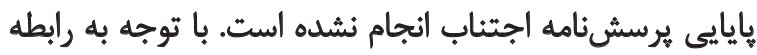

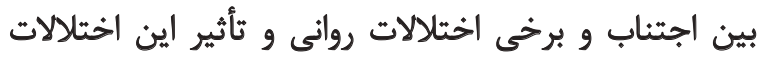

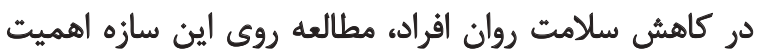

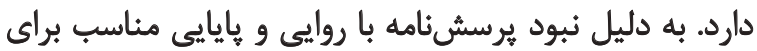

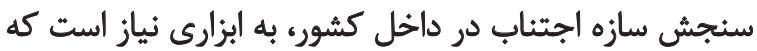

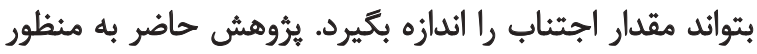

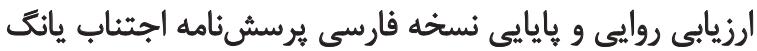

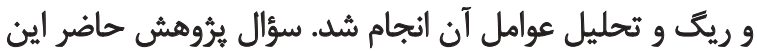

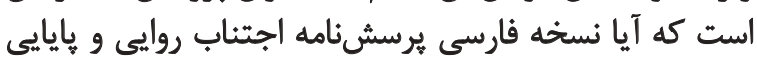

مناسبى دارد؟ مُو

مطالعه همبستگى حاضر از نوع مطالعات تحليل عوامل

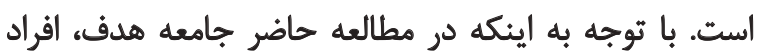

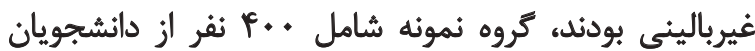

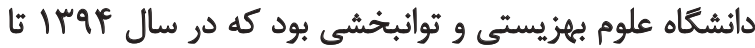

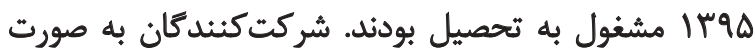

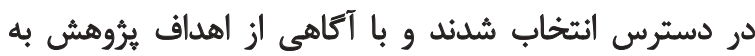

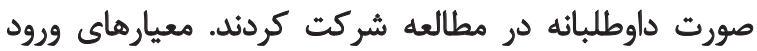

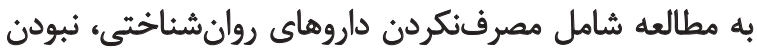

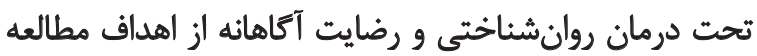

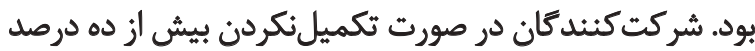

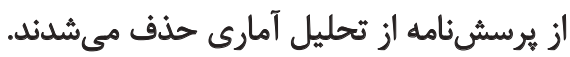

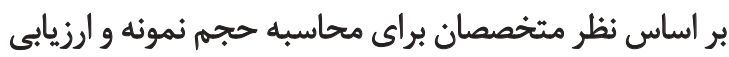

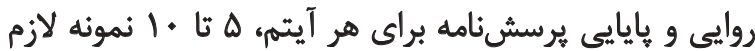

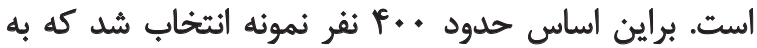

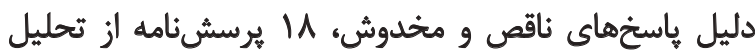

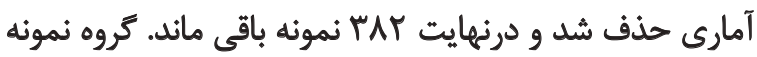

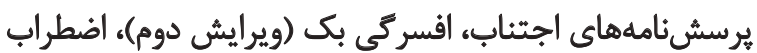

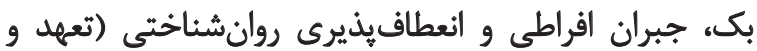

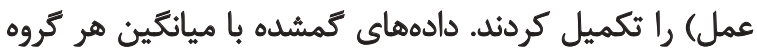

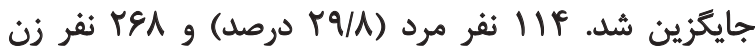

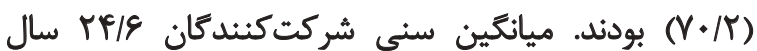

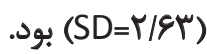

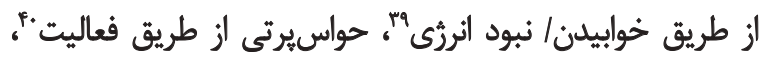

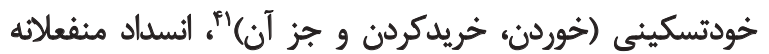

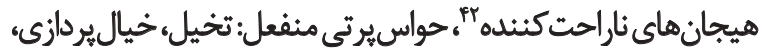

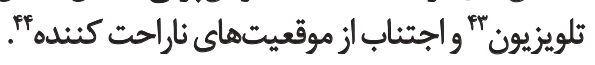

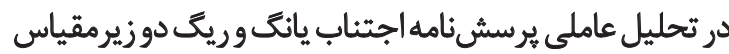

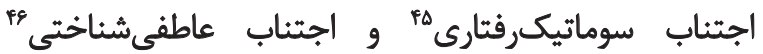

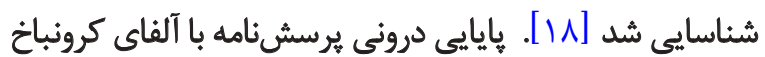

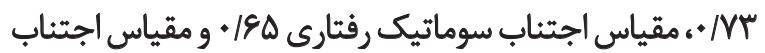

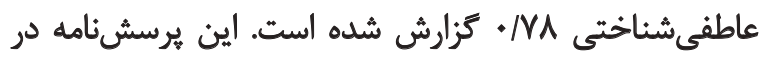

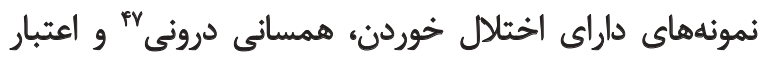

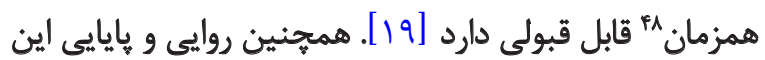

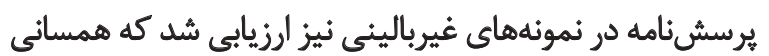

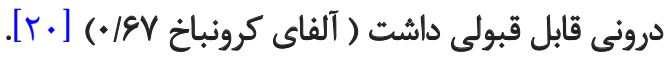

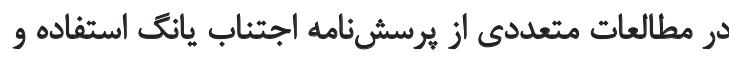

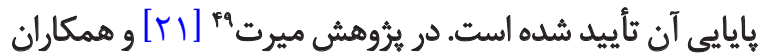

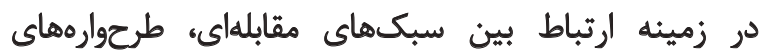
ناسازكار اوليه و اضطراب اجتماعى، ضرئ ضريب آلفاى زيرمقياس

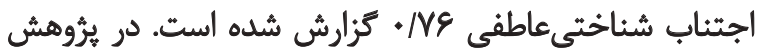

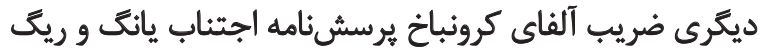

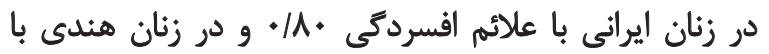

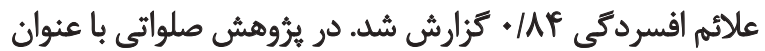

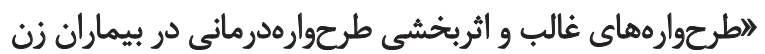

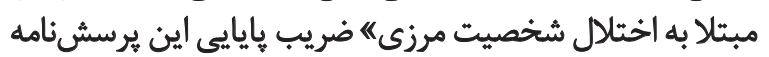

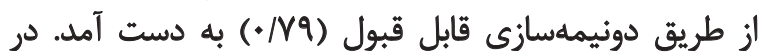

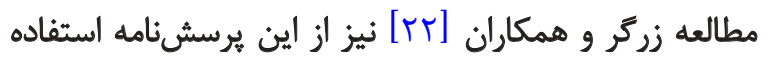

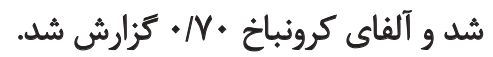
براساس ديدكاه نظرى طرحوارهدرمانى در تمامى انسان هايى

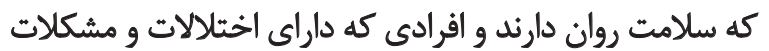

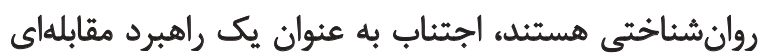

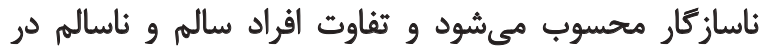

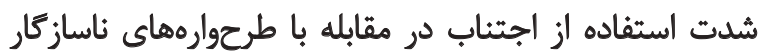

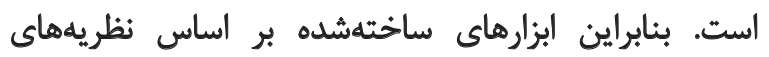

39. Avoidance through sleep/ lack of energy 40. Distraction through activity

41. Self-soothing (eating, shopping, etc.)

42. Passive blocking of upsetting emotions

43. Passive distraction: Fantasy, daydreaming, television 44. Avoidance of upsetting situations

45. Behavioral somatic avoidance

46. Cognitive emotional avoidance

47. Internal consistency

48. Concurrent validity

49. Meiret 
حاكى از تناسب باياييى و رواييى آن است. تحليل عاملى به روش

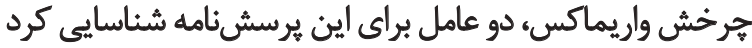

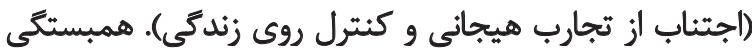

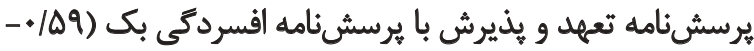

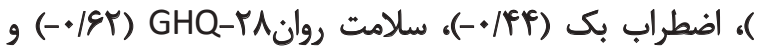

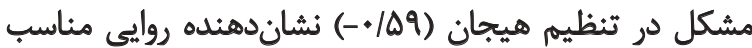

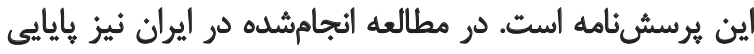

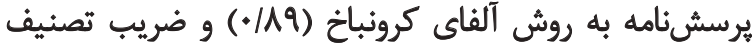

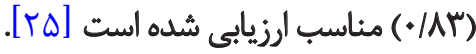

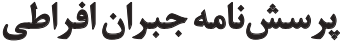

يرسش إنامه جبران افراطي را يائك در سال 1990 ساخت تابه

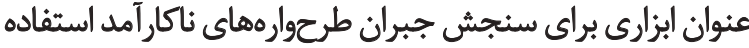

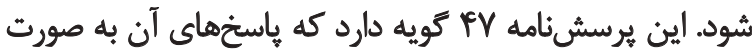

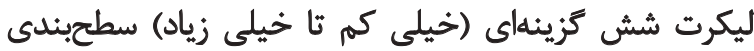

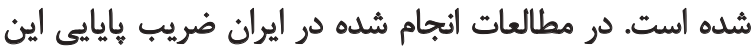

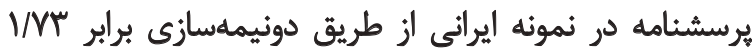

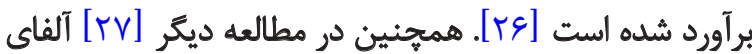

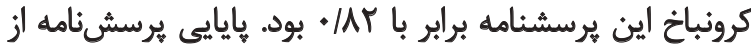

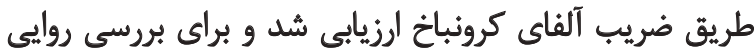

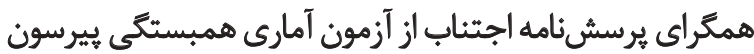

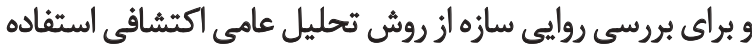

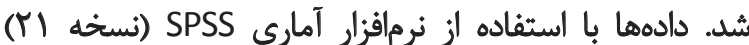

$$
\text { تجزيلوتحليل شد. }
$$

يافتهها

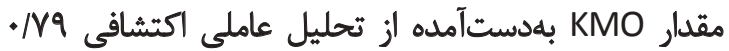

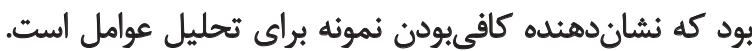

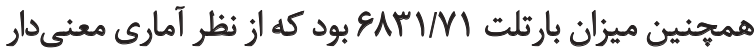

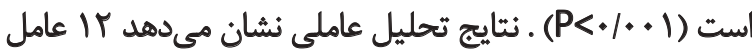

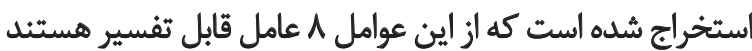

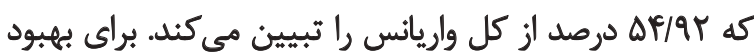

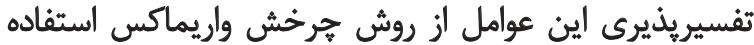

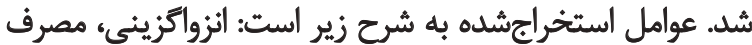

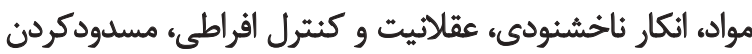

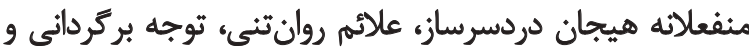

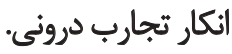

تعدادى از سوال هايیى كه ضريب عاملى كمتر از "َ/ • داشتند از

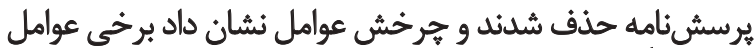

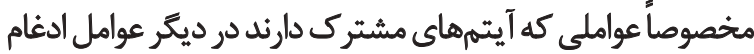

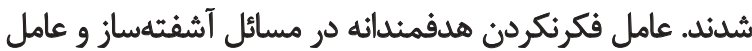

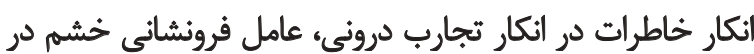

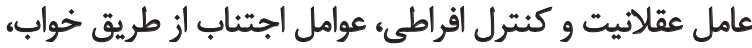

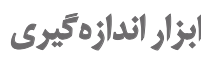

يرسش نامهاجتناب

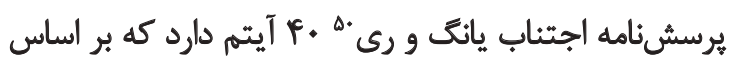

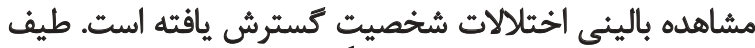

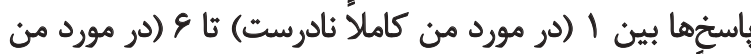

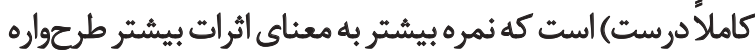

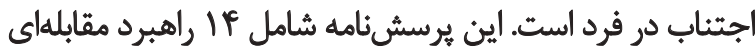

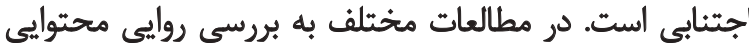

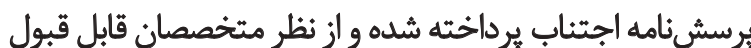

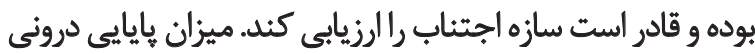

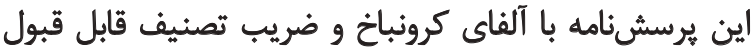
)

\section{برسش نامه افسردكى بك (نسخه دوم)}

بك و همكار انش اين مقياس رادر سال اعوال19 معرفى كردهاند

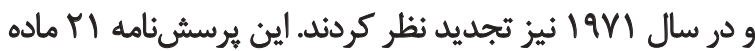

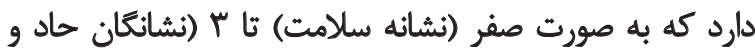

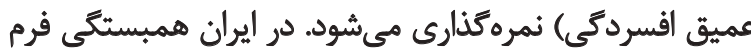

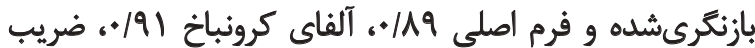

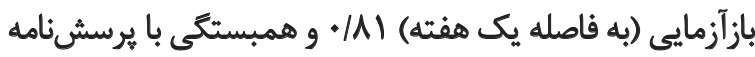
اضطراب بك اه|• كزارش شده است [بr].

\section{برسش نامه اضطراب بك}

اين يرسشنامه شامل اب كويه (ليكرت همار كزينهايى) است.

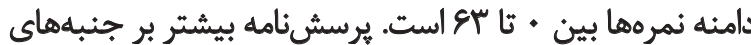

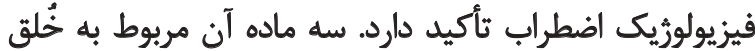

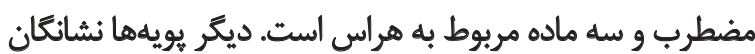
تنش حركتى را ميسنجند. بك و كلاركى (19A1) همسانى

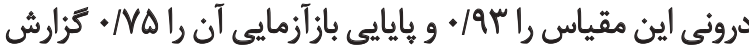

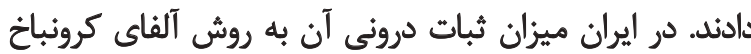

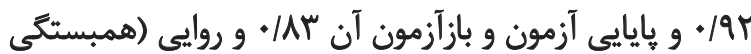

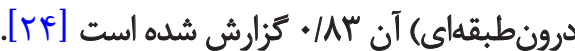

يرسشنامه هذيرش و عمل (نسخه دوم)

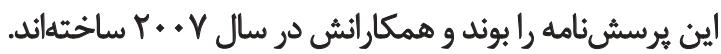

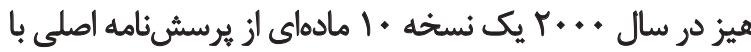

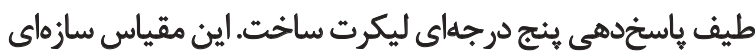

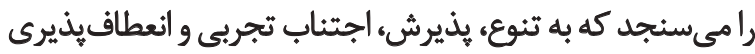

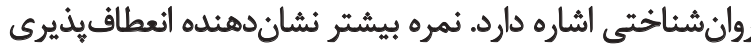

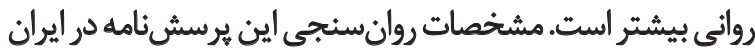

50. Young-Rygh Avoidance Inventory (YRAI) 
جدول ا. تويههاي مربوط به عوامل استخراجشده و بار عاملى هر تويه يس از جرخش واريماكس

\begin{tabular}{|c|c|c|}
\hline بار عاملى & عامل انزوآكزينى & \\
\hline . Ma & وقثتى احساساتم جريحهدار شود، از هردم كثارهيري مي كنم. & r. \\
\hline.$/ F \Delta$ & وقتى ناراحت هستم، از ديكران كنارهيرى مي كنه. & $r$. \\
\hline .194 & وقيتى عصبانى مي شوم، از جمع قاصله مي كيرم. & זו \\
\hline \multirow[t]{2}{*}{$\cdot / \mu r$} & وقتى خودم را مشغول كارى مي كنهى احساس ناراحتى نمى كنم. & $M$ \\
\hline & عامل مصرف مواد ع & \\
\hline .189 & براى خلاص شدن از شر عواطف ناخوشايند، به مشروب هناه ميرم. & $r$ \\
\hline$\cdot / \Delta r^{\circ}$ & براى دستيابى به آرامش و احساس بهتر، به دارو متوسل مىشوم. & $r$ \\
\hline \multirow[t]{2}{*}{$\cdot|A|$} & وقتى كه آشفته و عصبائى هستمه سيكار هي كشهر. & 9 \\
\hline & عامل انكار ثاخششنودى & \\
\hline$\cdot$ /AF & بهندرت احساس غمكينى و دل تنكى هي كنه. & r \\
\hline $.8 \%$ & سعى مي كثم به هساثل ناراحت كنثلده فكر نكثنه. & 1 \\
\hline \multirow[t]{2}{*}{.81} & ييشتر اوقات احساس شادى و خوشبختئي مي كنم. & r \\
\hline & عامل عقلاتيث و كثترل افراطي & \\
\hline$\cdot M$ & معتقدم بايد با استفاده از سلاح منطق، هيجان را كتترل كرد. & iv \\
\hline$\cdot M r$ & ن نمى توانم از كسى خيلى متنفر باشهي. & 《 \\
\hline .198 & اعتقاد راسخ من اين است كه اكر كارى أشتباه از آب در مى آيد، تاجايى كه امكان دارد بايد آن را فراموش كرد و به تلاش ادامه داد. & 19 \\
\hline \multirow[t]{2}{*}{$.18 \Delta$} & 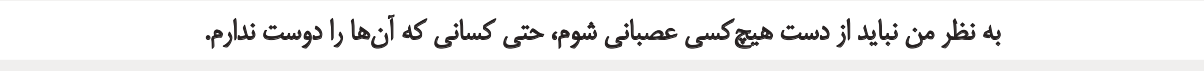 & $\varepsilon$ \\
\hline & عامل مسدودكردن منفعلانه هيجان دردسرساز & \\
\hline $.18 A$ & اغلب اوقات احساس خاصى نلارم، حتى مواقعى كه بلظاهر بسيار هيجانانكيز هستم. & "r \\
\hline .184 & 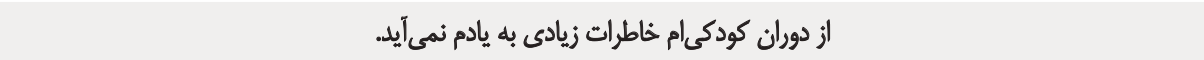 & r) \\
\hline \multirow[t]{2}{*}{$.19 x^{\infty}$} & 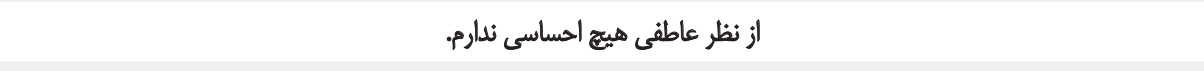 & 11 \\
\hline & عامل علائم روان تثنى & \\
\hline.$/ \mathrm{NA}$ & از مشكلات كوارشى (مثل سوء هاضمه، زخم معله، دردهاي شكمى) رنج مىبرم. & $1+$ \\
\hline$\cdot M$ & از دردهاي عضلانى رنج مىبرم. & 10 \\
\hline \multirow[t]{2}{*}{.NQ } & وقتى كارهايم بهخوبى ييش نمىرود، از نظر جسمى بيمار مى شوم. & ra \\
\hline & عامل توجهبر تردانى & \\
\hline. $\operatorname{ler}$ & براى اينكه احساس بهترى يييا كنم، وسايلى مىخرم كه درواقع به آنها نيازي ثلثارم. & re \\
\hline.$/ p r$ & اوقات زيادى را به خياليردازى هي كذارئم. & $r \Delta$ \\
\hline$. /{ }^{2} \mid$ & در طول روز خيلى هرت ميزنم يا مي خوابه. & $M$ \\
\hline \multirow[t]{2}{*}{.$/ M$} & 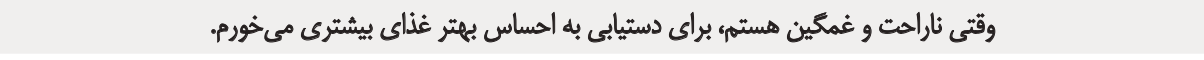 & re \\
\hline & عامل انكار تجارب درونى (خاطرات، احساسات و افكار) & \\
\hline .101 & سعى مى كثم به خاطرت تلخ و دردناك كذشته فكر نكثم. & rV \\
\hline$\cdot / \Delta r$ & سعى مى كثم به مسائل ناراحت كئده فكر نكثنم. & 1 \\
\hline.$/ 41$ & دوست ندارم به ناكامىها و كمبودهاي زندكى ام فكر كثم. & 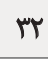 \\
\hline
\end{tabular}


جدول r. همبستّكى عوامل برسشنامه اجتناب با برسشئامه افسردكى، اضطراب، انعطاف روانشناختى و جبران افراطى

\begin{tabular}{|c|c|c|c|c|}
\hline انعطاف روانشُناختى & جبران اقراطى & الفسردكّى & اضطراب & عوسش استخرامه إجتئاب \\
\hline$+1+1$ &.$/ 1 V^{*}$ &.$/ M{ }^{*}$ &.$M a^{*}$ & انزواكزينى \\
\hline.$- / K q^{* * *}$ & $.1 \cdot 1$ & $.1+f$ &.$/ 4 *$ & مصرف مواد \\
\hline.$- / r \omega^{* *}$ & $\cdot \pi \cdot *$ &.$- /$ resen &.$- / 19 *$ & أنكار ناخشنودى \\
\hline$-\infty /$ &.$/ T v^{*}$ & $-\cdot / I^{*}$ & $-\cdot / r \cdot *$ & عقلانيت و كثترل افراطى \\
\hline$-* / \mathrm{N}^{*}$ &.$/ M I^{*}$ &.$- / 1 r$ &.$/ I^{*}$ & مسلوديردن منفعلانه هيجانات \\
\hline.$- / 48 * *$ &.$/ 1 E^{*}$ & $\cdot / r * *$ & $\cdot / r+*$ & علائم روانتنى \\
\hline$-* / \mu+\cdots$ & $. / 4+e=$ & $\cdot * / 4$ &.$/ 4 r^{*}$ & توجهبركردانى \\
\hline.$/ F^{*}$ & ( & -+111 & -+11 & انكار تجارب درونى \\
\hline$-\bullet / \mu \cdot *$ & $. / 4 r=*$ &.$- / I^{*}$ & $.4 a^{\circ}$ & نمره كل يرسش نامه اجتئاب \\
\hline
\end{tabular}

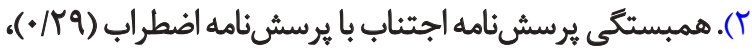

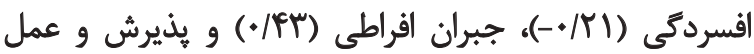

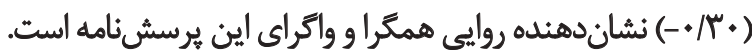

در جدول شماره س، ميزان همسانى درونى به روش آلفاى كرونباخ

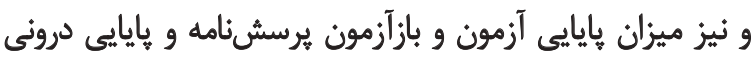

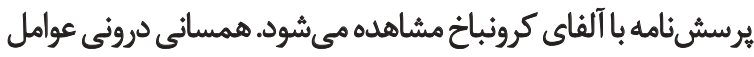

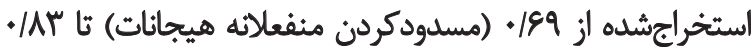

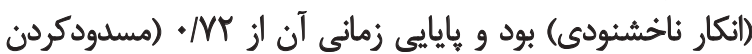

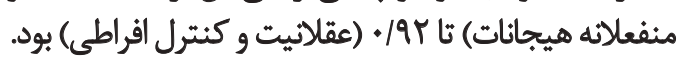

خودآرامبخشي و توجهبركر داني منفعلانه در عامل توجهبركَ داني

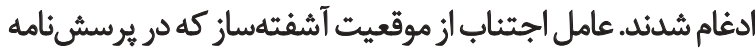

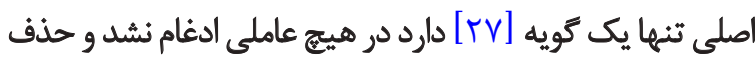

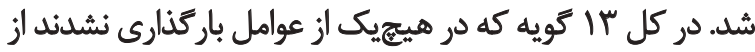
تحليل آمارى حذف شد. در جدول شماره ا بار عاملي هر هر مويه مشاهله مي شُود. براي ارزيابى روايي همكرا و واكراي برسشنامه اجتناب ازئ

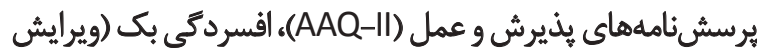

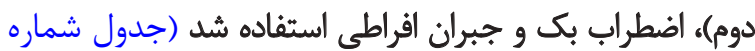

جدول ז. همسائى درونى و هايايى زمائى عوامل يرسشئامه اجتناب

\begin{tabular}{|c|c|c|}
\hline إيائي زمانى & همسائى درونى & عوامل استخر اجشده يرسش ثامه اجتئاب \\
\hline$\cdot / M$ & $\cdot|A|$ & انزواكزيني \\
\hline$\cdot N 9$ & $\cdot / A+$ & مصرف مواد \\
\hline.$/ 91$ & ./Ar & 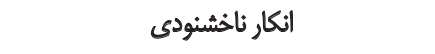 \\
\hline.$/ 94$ & ./Na & عقالانيت و كنترل افراطى \\
\hline$\cdot M$ & .189 & هسلوديردن منفعلانه هيجاناتات \\
\hline - /Af & $\cdot M^{e}$ & علاثم روانتئى \\
\hline$\cdot / M$ & $\cdot M$ & توجهبركر دائي \\
\hline . $\mathrm{NA}$ & - Ne & أنكار تجارب درونى \\
\hline$\cdot / A r$ & $\cdot / \mathrm{Na}$ & نمره كل يرسش نامه اجتناب \\
\hline
\end{tabular}




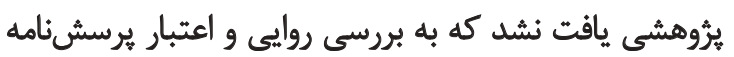

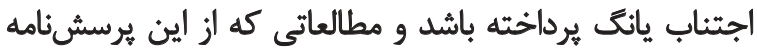

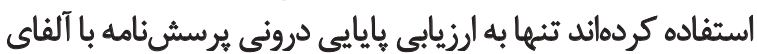

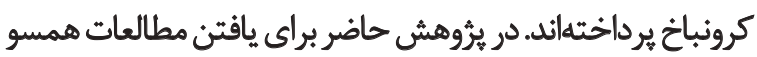

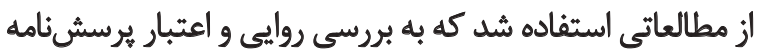

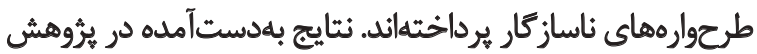

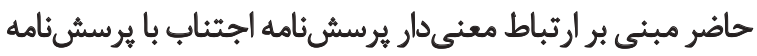

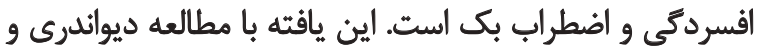

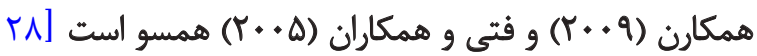

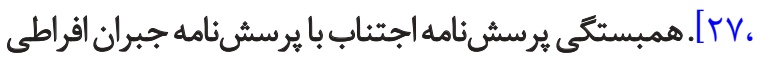

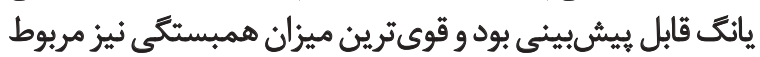

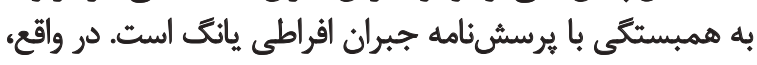

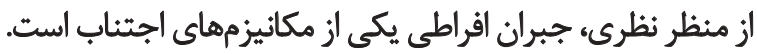

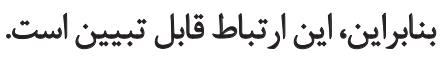

تثيجهيرى

نتايج مطالعه حاضر نشان مى دهد نسخه فارسى برسش نائنامه

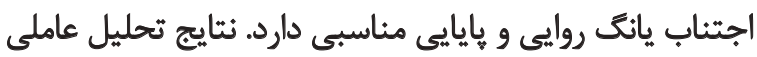

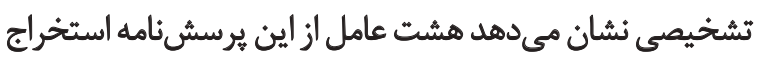

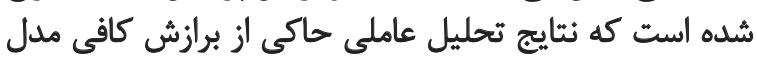

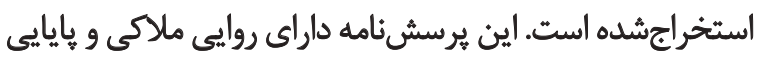

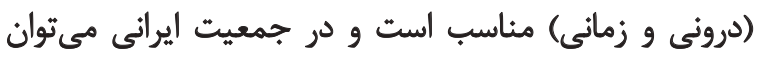

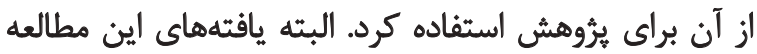

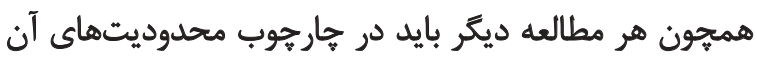

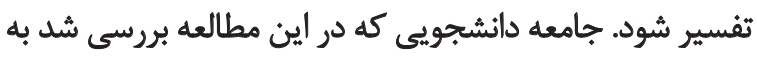

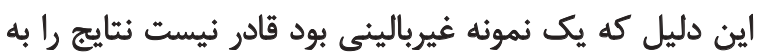
كروه بالينى تعميم دهد.

يكى از محدوديتهاي اين يُروهش استفاده از نمونه آمارى برى بردي

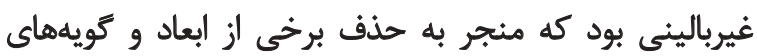

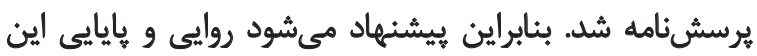

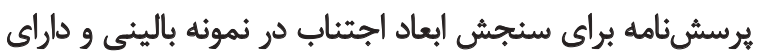

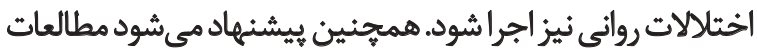

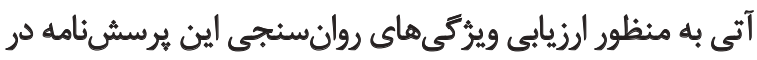
جمعيت بالينى انجام شود. سباسكَّزارى محققان بر خود لازم مي اناند از مسئولان محترم كميته

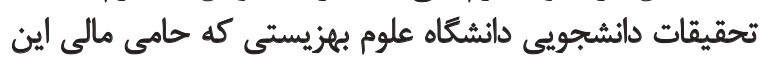

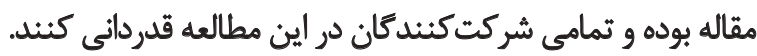

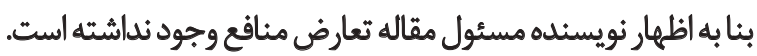

حاضس از بررسى تحليل عوامل يرسشنامه اجتناب در مطالعه

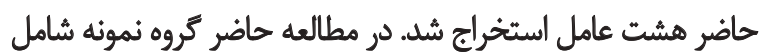

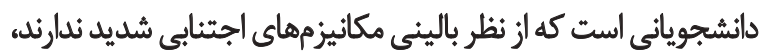

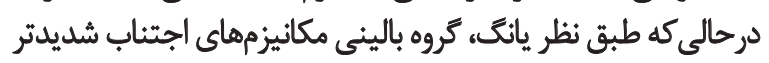

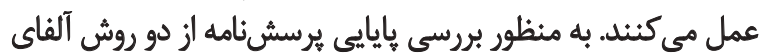

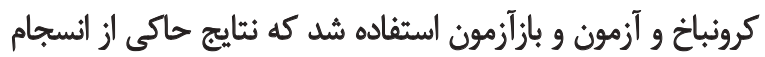

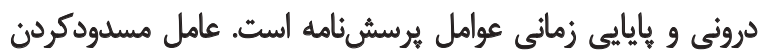

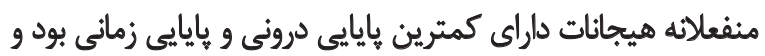

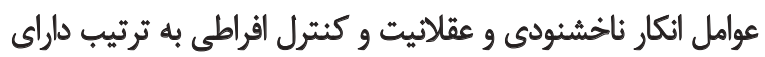

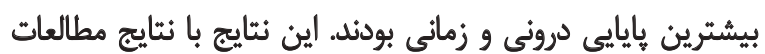

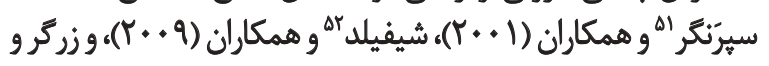

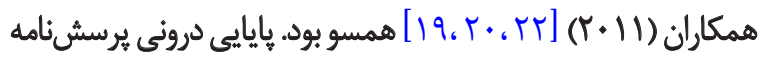

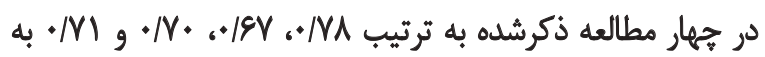

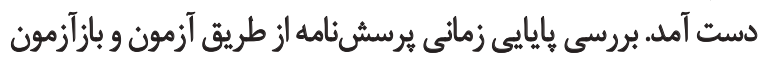

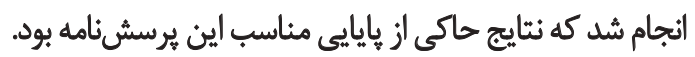

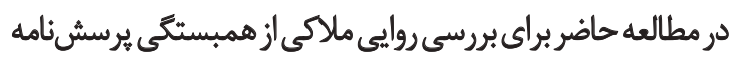

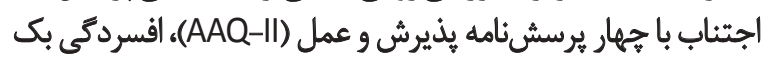

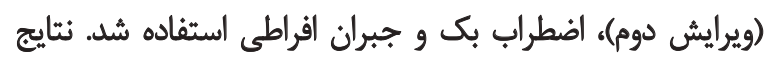

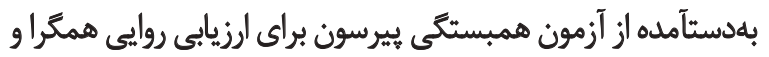

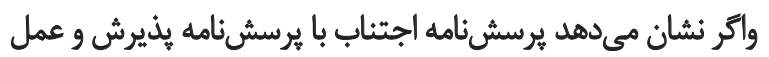

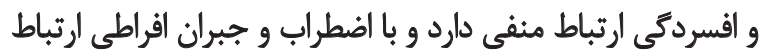

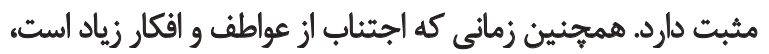

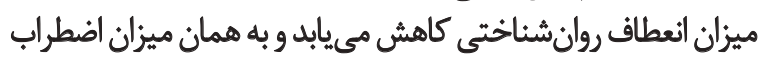

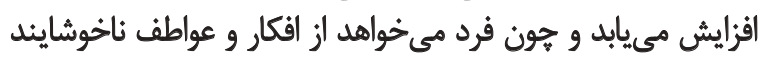

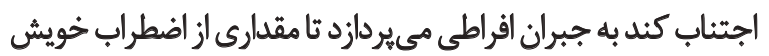

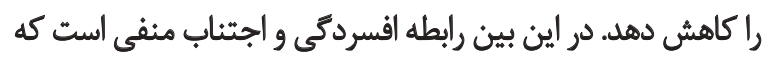

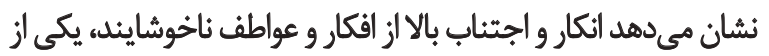

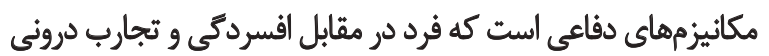

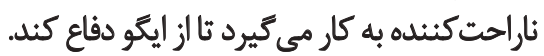

در تبيين اين يافته مىثوان به اين نكته اشاره كرد كه سازه

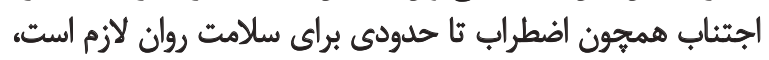

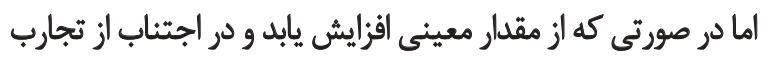

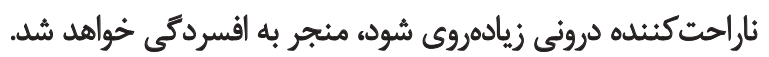

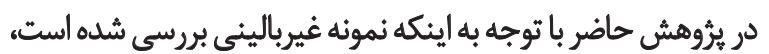

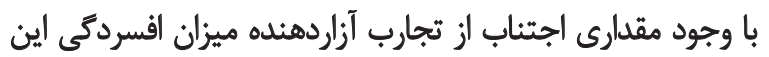

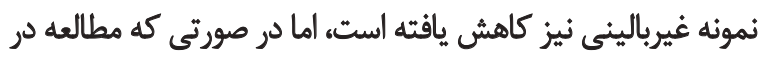

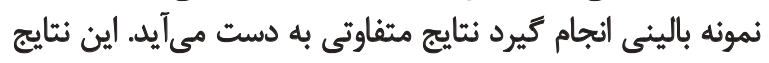

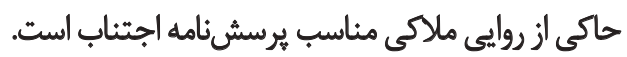




\section{References}

[1] Kahl KG, Winter L, Schweiger U. The third wave of cognitive behavioural therapies. Current Opinion in Psychiatry. 2012; 25(6):522-8. doi: 10.1097/yco.0b013e328358e531

[2] Fusco GM, Freeman A. Borderline personality disorder: A patient's guide to taking control. New York: WW Norton \& Company; 2004.

[3] Young JE, Klosko JS, Weishaar ME. Schema therapy: A practitioner's guide. New York: Guilford Press; 2003.

[4] Sempértegui GA, Karreman A, Arntz A, Bekker MHJ. Schema therapy for borderline personality disorder: A comprehensive review of its empirical foundations, effectiveness and implementation possibilities. Clinical Psychology Review. 2013; 33(3):426-47. doi: 10.1016/j.cpr.2012.11.006

[5] De Andrés RD, Aillon N, Bardiot M-C, Bourgeois P, Mertel S, Nerfin F, et al. Impact of the life goals group therapy program for bipolar patients: An open study. Journal of Affective Disorders. 2006; 93(1-3):253-7. doi: 10.1016/j.jad.2006.03.014

[6] Haghighat Manesh E, Baf AM, Reza H, Ghanbari Hashem Abadi BA, Mahram B. [Early maladaptive schemas and schema domains in rapists (Persian)]. Iranian Journal of Psychiatry and Clinical Psychology. 2010; 16(2):145-53.

[7] Moosaviassl SA, Moosavi Sadat Z. [The effectiveness of schema therapy in reduction of early maladaptive schemas on PTSD veterans men (Persian)]. Armaghane Danesh. 2014; 19(1):89-99.

[8] Ottenbreit ND, Dobson KS. Avoidance and depression: The construction of the cognitive-behavioral avoidance scale. Behaviour Research and Therapy. 2004; 42(3):293-313. doi: 10.1016/s00057967(03)00140-2

[9] Lawson R, Waller G, Lockwood R. Cognitive content and process in eating-disordered patients with obsessive-compulsive features. Eating Behaviors. 2007; 8(3):305-10. doi: 10.1016/j.eatbeh.2006.11.006

[10] Kashdan TB, Barrios V, Forsyth JP, Steger MF. Experiential avoidance as a generalized psychological vulnerability: Comparisons with coping and emotion regulation strategies. Behaviour Research and Therapy. 2006; 44(9):1301-20. doi: 10.1016/j. brat.2005.10.003

[11] Lavy EH, van den Hout MA. Cognitive avoidance and attentional bias: Causal relationships. Cognitive Therapy and Research. 1994; 18(2):179-91. doi: 10.1007/bf02357223

[12] Feldner M., Zvolensky M., Eifert G., Spira A. Emotional avoidance: An experimental test of individual differences and response suppression using biological challenge. Behaviour Research and Therapy. 2003; 41(4):403-11. doi: 10.1016/s0005-7967(02)00020-7

[13] Kämpfe CK, Gloster AT, Wittchen HU, Helbig-Lang S, Lang T, Gerlach AL, et al. Experiential avoidance and anxiety sensitivity in patients with panic disorder and agoraphobia: Do both constructs measure the same. International Journal of Clinical and Health Psychology. 2012; 12(1):5.

[14] Lee JK, Orsillo SM, Roemer L, Allen LB. Distress and avoidance in generalized anxiety disorder: Exploring the relationships with intolerance of uncertainty and worry. Cognitive Behaviour Therapy. 2009; 39(2):126-36. doi: 10.1080/16506070902966918
[15] Orsillo SM. Acceptance and commitment therapy in the treatment of posttraumatic stress disorder. Behavior Modification. 2005; 29(1):95-129. doi: 10.1177/0145445504270876

[16] Begotka AM, Woods DW, Wetterneck CT. The relationship between experiential avoidance and the severity of trichotillomania in a nonreferred sample. Journal of Behavior Therapy and Experimental Psychiatry. 2004; 35(1):17-24. doi: 10.1016/j. jbtep.2004.02.001

[17] Young JE. Cognitive therapy for personality disorders: A schema-focused approach. Fruitville, Florida: Professional Resource Press; 1994

[18] Luck A, Waller G, Meyer C, Ussher M, Lacey H. The role of schema processes in the eating disorders. Cognitive Therapy and Research. 2005; 29(6):717-32. doi: 10.1007/s10608-005-9635-8

[19] Spranger SC, Waller G, Bryant-Waugh R. Schema avoidance in bulimic and non-eating-disordered women. International Journal of Eating Disorders. 2001; 29(3):302-6. doi: 10.1002/eat.1022

[20] Sheffield A, Waller G, Emanuelli F, Murray J, Meyer C. Do schema processes mediate links between parenting and eating pathology. European Eating Disorders Review. 2009; 17(4):290300. doi: $10.1002 /$ erv.922

[21] Mairet K, Boag S, Warburton W. How important is temperament? The relationship between coping styles, early maladaptive schemas and social anxiety. International Journal of Psychology and Psychological Therapy. 2014; 14(2):171-90.

[22] Zargar M, Kakavand AR, Jalali MR, Salavati M. [Comparison of maladaptive early schemas and avoidance behaviors in opioid dependent men and non-dependent men (Persian)]. Journal of Applied Psychology. 2011; 5(1):69-84.

[23] Stefan-Dabson K, Mohammadkhani P, Massah-Choulabi O. [Psychometrics characteristic of Beck Depression Inventory-II in patients with magor depressive disorder (Persian)]. Journal of Rehabilitation. 2007; 8:82-0.

[24] Kaviani H, Mousavi AS. [Psychometric properties of the Persian version of Beck Anxiety Inventory (BAI) (Persian)]. Tehran University Medical Journal. 2008; 66(2):136-40.

[25] Abasi E, Fti L, Molodi R, Zarabi H. [Psychometric properties of Persian version of acceptance and action questionnaire-II (Persian)]. Journal of Psychological Models and Methods. 2013; 3(10):65-80

[26] Bayrami M, Bakhshipor A, Esmaeili A. The relationship between coping styles and early maladaptive schemas in disconnection-rejection and over vigilance-inhibition in young's schema model. Journal of Life Science and Biomedicine. 2012; 2(4):178-81.

[27] Noorbala F, Bahram Ehsan H, Alipour A. [Predictive effects of avoidance and overcompensation coping styles on general health (Persian)]. Health Psychology. 2016; 5(17):51-63.

[28] Divandari H, Ahi Gh, Akbari H, Mahdian H. [The young Schema Questionnaire - Short Form (SQ-SF): Investigation psychometric properties and factor structure between students In Islamic Azad University, Kashmar Branch (Persian)]. PazhouheshNameye Tarbiati. 2009; 5(20):103-133.

[29] Fata L, Birashk B, Atefvahid MK, Dabson KS. Meaning assignment structures/schema, emotional states and cognitive processing of emotional information: comparing two conceptual frameworks. Iranian Journal of Psychiatry and Clinical Psychology. 2005; 11(3):312-26. 
